DOI - https://doi.org/10.5965/2316796309182020150

\title{
ABORDANDO AS INTER-RELAÇÕES ENTRE USUÁRIO IDOSO E AMBIENTE DOMÉSTICO PELA ÓTICA DOS FATORES HUMANOS
}

\section{ADDRESSING THE INTERRELATIONS BETWEEN THE ELDERLY AND THE DOMESTIC ENVIRONMENT FROM THE PERSPECTIVE OF HUMAN FACTORS}

Maria de Lourdes Capponi Arruda Koehler ${ }^{1}$ Milton José Cinelli 2 Susana Cristina Domenech 3 


\section{Resumo}

Operfildapopulaçãomundialvemsofrendo um processo gradual de envelhecimento. Compreender as capacidades e limitações dos idosos é fundamental nos projetos, o que implica no entendimento do processo de envelhecimento e dos desafios que o ambiente construído pode representar ao usuário. Desse modo, o objetivo do estudo foi, mediante uma revisão de literatura, identificar quais as relações entre idosos e ambiente doméstico, principalmente no que se refere aos fatores de riscos físicos. Os resultados possibilitam inferir que 0 estudo sobre a relação pessoa-ambiente construído pode trazer relevantes contribuições para o processo de avaliação dos riscos domésticos e modificações no ambiente. Built Environment; Domestic risk factors. Ambiente construído; Fatores de risco domésticos.

Abstract

The profile of the world population has been undergoing a gradual aging process. limitations of the elderly is important in projects, which implies the understanding of the aging process and the challenges that the built environment can represent to the user. Thus, the aim of the study was, through a literature review, to identify the elationships between the elderly and the home environment, especially the ones involving physical risk factors. The results make it possible to infer that the study on can bring significant contributions to the evaluation process of domestic risks and changes in the environment.

Key-words: Elderly; Home; Ergonomics; Understanding the capabilities and the person-built environment relationship

${ }^{1}$ Mestra - UDESC (marylu.igu@gmail.com)

\section{INTRODUÇÃO}

Estamos em uma época na qual, é cada vez mais evidente a mudança sem precedentes da demografia, ocasionando que vários autores debatam sobre esta preocupação, sobretudo no que tange ao envelhecimento da população. Uma população que apresenta uma esperança média de vida que tende a ser cada vez maior do que já tenha sido anteriormente registado, e consequentemente, gerando um fator que diretamente tem implicações na abordagem do design (PREISER, 2001).

O papel do ambiente construído doméstico, para manter e melhorar a função diária dos adultos mais velhos, é agora amplamente reconhecido na pesquisa e na prática. Várias áreas da ciência enfatizaram este tipo de ambiente como um fator objetivo crítico que sustenta ou prejudica o funcionamento individual, principalmente no caso dos indivíduos mais velhos (WAHL et al., 2009). Conforme estes mesmos autores, a avaliação do papel do ambiente doméstico no processo de incapacitação progressiva decorrente do envelhecimento contínua sendo uma importante preocupação de saúde pública, dado que os adultos mais velhos preferem "envelhecer no local" o maior tempo possível. Estudos populacionais sugerem que os ganhos funcionais em indivíduos idosos nas últimas décadas podem ser atribuídos em parte à redução de barreiras ambientais, também conhecidas como perigos domésticos e riscos ambientais (WAHL et al., 2009).

Segundo Kruse et al. (2010) aproximadamente metade de todas as quedas ocorrem dentro e ao redor das residências das pessoas; essa proporção excede $60 \%$ para adultos com 80 anos ou mais. E metade de todas estas quedas envolvem algum tipo de fator de risco ambiental. Assim, melhorar a segurança física das famílias pode ser uma estratégia para reduzir quedas e outros tipos de acidentes domésticos. Os autores prosseguem dizendo que, cada vez mais, está sendo reconhecida, a importância de fazer mudanças no ambiente doméstico de forma a permitir que as pessoas envelheçam com segurança em um ambiente que Ihe seja familiar.

Portanto, este estudo teve como ponto de partida uma revisão sistemática realizada com o objetivo de identificar, junto à literatura qualificada, estudos referentes a relação entre os idosos e o ambiente construído domiciliar, principalmente no que se refere aos fatores de riscos físicos. Buscou-se identificar artigos e outros materiais que pudessem servir de embasamento e que auxiliassem a responder as seguintes questões: Quais são as evidências que apoiam uma relação entre os ambientes construídos domésticos inadequados e os fatores de risco relacionados as debilidades decorrentes do envelhecimento? E quais são as evidências sobre os efeitos das modificações no ambiente domiciliar sobre os resultados relacionados a diminuição dos acidentes domésticos decorrentes dos fatores de risco? Especificamente, buscou-se conhecer o delineamento dos estudos, quais as linhas metodológicas utilizadas e os resultados por eles obtidos. E, desta maneira, desenvolver uma melhor compreensão das interrelações entre as pessoas idosas e seus ambientes físico-sociais e como essas mesmas inter-relações influenciam as atividades diárias das pessoas mais velhas.

\section{REFERENCIAL TEÓRICO}

\subsection{ENVELHECIMENTO}

O envelhecimento populacional é um fenômeno mundial e o Brasil encontra-se 
entre os países da América Latina que têm experimentado maior aumento no número de pessoas da chamada terceira idade.

Envelhecer é o processo de perda física e mental que acontece com todas as pessoas não atingidas por doenças fatais. Spirduso $(2005$, p.6), conceitua o termo envelhecimento como "um processo ou conjunto de processos que ocorrem em organismos vivos e que com o passar do tempo levam a uma perda de adaptabilidade, deficiência funcional, e, finalmente, à morte". Então, segundo a definição do autor o envelhecimento é uma extensão lógica dos processos fisiológicos do crescimento e desenvolvimento do ser humano, porém, é diferente a taxa na qual os sistemas fisiológicos e comportamentais envelhecem dentro do indivíduo.

O processo de envelhecimento naturalmente promove modificações no corpo. Ao chegar à chamada terceira idade, as pessoas se deparam com uma diminuição da massa óssea e muscular, com uma perda gradativa da força física e de flexibilidade. Isto sem contar o envelhecimento do sistema nervoso que altera não apenas o aspecto físico mas também, causa transtornos de ordem comportamental. Devido a estas perdas, há uma redução do alcance funcional e da flexibilidade dos movimentos, especialmente nos membros (SPIRDUSO, 2005). Além disso, para muitos idosos há também a questão da autoconsciência do seu lugar perdido na sociedade. Os aposentados passam a fazer parte de um grupo social diferente, que os separa da classe ativa e produtora. Se o idoso continua ativo na sociedade, mantendo sua autoestima, é considerado "saudável" pelos estudiosos (BESTETTI, 2006)

\subsection{O IDOSO E A MORADIA}

Segundo a OMS (2008, p. 30) a "moradia é fundamental para a segurança e bem-estar". E que "há uma relação direta entre uma moradia apropriada e acesso a serviços comunitários e sociais que influenciam a independência e a qualidade de vida dos idosos". As pessoas sofrem com o avançar da idade, as alterações anatômicas são inevitáveis e este aspecto é imprescindível na compreensão de como o espaço interage com o idoso. O espaço deve inspirar confiança e segurança, ou seja, o conhecimento dos ambientes em seus pequenos detalhes é um apelo muito forte no sentido de trazer tranquilidade interna e identidade (HAZIN, 2012)

Segundo Stamato (2007), ambiente construído onde o usuário idoso está inserido é um fator fundamental no processo de autonomia e independência. O oferecimento de ambientes adaptados de acordo com as dificuldades, deficiências ou incapacidades dos indivíduos que os frequentam, irá favorecer a manutenção da autonomia e independência destes mesmos indivíduos. Darè (2006) afirma que o desgaste natural do envelhecimento dificulta o desempenho das tarefas diárias, obrigando os idosos a se relacionarem com um mobiliário que já não lhes é satisfatório do ponto de vista físico. Dessa forma, as pessoas vão forçando, no dia a dia, uma adaptação que na maioria das vezes trazem lesões somente percebidas posteriormente.

\subsection{OS ACIDENTES E OS RISCOS DOMESTICOS}

A queda é um evento preocupante na vida dos idosos e, por vezes, com consequências muito sérias. As quedas são consideradas os acidentes domésticos mais comuns entre pessoas idosas no mundo todo (FONG et al., 2015). Segundo Urton (1991) as quedas seriam as causadoras da maioria das lesões fatais e não fatais em adultos com mais de 65 anos, bem com a maior causa de morte por lesão nas pessoas idosas. E afirma que as quedas e suas sequelas podem ter um grande impacto na autoconfiança e independência dos idosos. Esta perda de autoconfiança poderia ocasionar uma diminuição da mobilidade, com uma consequente diminuição na qualidade de vida e um aumento da debilidade geral do idoso, o que acarretaria um aumento da necessidade de serviços de saúde (URTON, 1991).

Acredita-se que as quedas não sincopais resultem de combinação de fatores intrínsecos e extrínsecos. Os fatores de risco intrínsecos incluem idade avançada, artrite acidente vascular cerebral, problemas nos pés, uso de medicações e deficiências na cognição, força muscular, equilíbrio, marcha e visão (GILL; WILLIAMS; TINETTI, 2000). Os fatores extrínsecos estão relacionados aos riscos domésticos, como quando os idosos utilizam mobiliário doméstico inadequado e mal posicionado para realizarem determinadas tarefas, ou mesmo ao adotarem hábitos perigosos como subir em escadas móveis para alcançar armários altos (MORGAN et al., 2005; ROMLI et al., 2018; STEVENS; HOLMAN; BENNETT, 2001).

Os riscos domésticos não são apenas definidos pelas características físicas do ambiente, mas são produtos dos riscos encontrados pelo indivíduo durante a realização de atividades funcionais no ambiente doméstico. No entanto, investigar os riscos domésticos como um fator independente, sem levar considerar a interação das pessoas idosas com o ambiente doméstico pode levar a resultados inconsistentes. Pois, essa abordagem unilateral não leva em conta as características dos indivíduos mais velhos e como isso ocorre. podendo tornar o ambiente doméstico ainda mais perigoso (ROMLI et al., 2018).

\subsection{O IDOSO, O AMBIENTE E OS FATORES HUMANOS}

Tal como afirmou Simões (2006), todos nós, em diversos momentos da nossa vida, experimentamos dificuldades nos espaços em que vivemos ou com os produtos e serviços que usamos. Estas dificuldades resultam de situações de inadaptação das características do meio construído ou dos produtos e serviços face às nossas necessidades. Porém, fazemos parte de uma sociedade onde os espaços são desenhados para um homem médio, ausente de incapacidades, o que é uma falácia, pois além de sermos todos diferentes, nossa população tender a ser cada vez mais idosa e portadora de alguma incapacidade, seja ela física, psicológica ou sensorial, e, consequentemente, com necessidades diversas. Fatos estes que tornam os espaços projetados para um homem mítico cada vez mais inadequados. Assim, torna-se imperativo projetar espaços capazes de satisfazer todas as necessidades dos seus utilizadores, bem como, descobrir soluções que satisfaçam a todos, independentemente da sua idade e limitações.

Segundo Jacko et al. (2012), uma das principais preocupações para os fatores humanos/ergonomia reside na capacidade de fazer generalizações e previsões sobre as interações humanas para melhorar a produtividade, segurança e satisfação geral do usuário. Pela própria natureza de sua origem, os fatores humanos/ergonomia são um campo interdisciplinar de estudos compreendendo aspectos da psicologia, fisiologia, engenharia, estatística, ciência da computação, e outras ciências físicas e sociais. Aspecto este que é muito importante pois, ao estudar a relação entre o envelhecimento e o ambiente doméstico, não é adequado medir apenas aspectos objetivos da habitação, como barreiras ambientais físicas e acessibilidade, é necessário, também, observar os aspectos percebidos da habitação (KYLÉN et al., 2014)

HFD, v.9, n 18, p. 150-170, dezembro 2020 


\section{METODOLOGIA}

Este estudo teve como ponto de partida a realização de uma revisão sistemática de literatura. De acordo com Conforto, Amaral e Silva (2011), a revisão sistemática é um método científico para a busca e análise de artigos de uma determinada área da ciência. Ou seja, busca-se compreender o "estado da arte" do assunto pesquisado. Objetiva agrupar e expor informações significativas encontradas em relação a uma questão, produzindo sínteses de resultados vindos de diferentes fontes (FELIZARDO et al, 2017). Assim, o presente artigo utilizou o método proposto por Conforto, Amaral e Silva (2011) para a realização desta revisão sistemática.

Desta forma, o passo inicial foi estabelecer um protocolo contendo informações relevantes para o desenvolvimento da pesquisa (Quadro 1).

Quadro 1 - Resumo do protocolo da Revisão Sistemática de Literatura (RSL).

\begin{tabular}{|c|c|}
\hline \multicolumn{2}{|c|}{ RESUMO DO PROTOCOLO DA REVISÃO SISTEMÁTICA DE LITERATURA } \\
\hline Objetivo & $\begin{array}{l}\text { Identificar, junto à literatura qualificada, pesquisas } \\
\text { referentes a inter-relação entre os idosos e o ambiente } \\
\text { construído doméstico, principalmente no que se } \\
\text { refere aos fatores de riscos físicos. }\end{array}$ \\
\hline Palavras-Chave & $\begin{array}{l}\text { - Elderly: Elder, Elderly, Elder*,Old People, Older, } \\
\text { Aged, Aging; } \\
\text { - Idosos: Idoso, Idosos, Senescentes; } \\
\text { - Environmental Built: Environmental Built, Housing. } \\
\text { Home; } \\
\text { - Ambiente construído: Ambiente construído, } \\
\text { Moradia, Casa; } \\
\text { - Environmental risk: Environmental risk, falls, } \\
\text { accidental falls, home hazards; } \\
\text { - Riscos domésticos: Riscos domésticos, quedas, } \\
\text { risco de quedas. }\end{array}$ \\
\hline $\begin{array}{l}\text { Operadores } \\
\text { booleanos }\end{array}$ & $\begin{array}{l}\text { and, or, not (foram utilizados respeitando as } \\
\text { diferenças entre as bases de dados). }\end{array}$ \\
\hline $\begin{array}{l}\text { Fontes de } \\
\text { busca }\end{array}$ & Scopus - Elsevier e Web of Science - WoS. \\
\hline $\begin{array}{l}\text { Período da } \\
\text { busca }\end{array}$ & No mês de abril de 2018. \\
\hline $\begin{array}{l}\text { Critérios de } \\
\text { inclusão }\end{array}$ & $\begin{array}{l}\text { - Publicações nacionais e internacionais acessíveis de } \\
\text { forma gratuita através do Portal de Periódicos da } \\
\text { CAPES, Google Acadêmico ou portal das editoras; } \\
\text { - Estudos incluindo artigos de periódicos, artigos de } \\
\text { congressos, livros, capítulos de livros, manuais, } \\
\text { normas técnicas; } \\
\text { - Estudos escritos em Inglês, Português ou Espanhol; } \\
\text { - Estudos realizados a partir de } 1980 \text { até junho de } \\
\text { 2018. }\end{array}$ \\
\hline $\begin{array}{l}\text { Estratégia de } \\
\text { seleção }\end{array}$ & $\begin{array}{l}\text { - Filtro 1: leitura de título, resumo e palavras-chave; } \\
\text { - Filtro 2: leitura completa dos artigos. }\end{array}$ \\
\hline
\end{tabular}

Fonte: Elaborado pelos autores (2018)
Como se pode observar, foram incluídas publicações nacionais e internacionais, disponíveis nos idiomas português, espanhol e inglês, e que abordassem temas relativos à avaliação da inter-relação entre o usuário idoso e o ambiente construído doméstico, bem como os fatores de risco.

Sendo assim, com base no protocolo estabelecido, realizou-se a pesquisa. Foram encontrados, nas bases de dados pesquisadas, um total de 508 trabalhos, sendo 301 na SCOPUS e 207 na WoS. Destes 508, foram desconsiderados trabalhos repetidos e/ ou não encontrados, no total de 62 publicações. Desta forma, 446 foram relacionados para o primeiro filtro, que consistiu em ler todos os títulos, resumos e palavras chave para verificar se realmente continham os descritores correspondentes, e, também, se tinham relação com o tema abordado. Isto resultou na exclusão de 392 artigos. Ou seja, 54 trabalhos foram selecionados para a aplicação do segundo filtro.

A segunda filtragem consistiu na leitura completa dos artigos com o objetivo de compreender como os autores trataram do tema pesquisado. O que resultou em 14 trabalhos considerados válidos por trazerem contribuições significativas para o tema proposto. A síntese da busca e seleção de artigos pode ser visualizada na figura 1.

Figura 1 - Resultado das buscas e da aplicação dos filtros

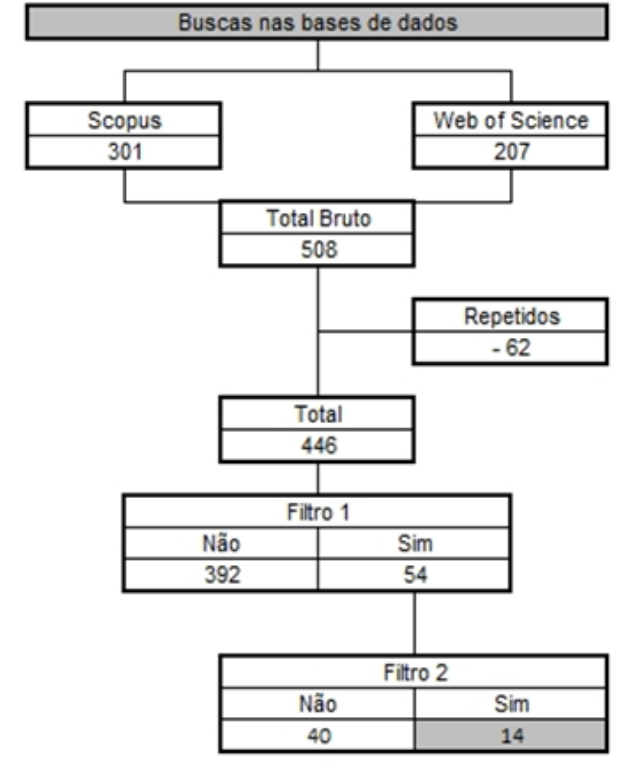

Fonte: Elaborado pelos autores (2018)

\section{RESULTADOS}

Apesar do grande número de artigos iniciais (508), poucos puderam ser elencados (14). Porem, os resultados foram bastante relevantes: pôde-se elencar diversos autores que tratam da temática, encontrar fundamentações teóricas bastante sólidas e aplicações de critérios de avaliação de risco físico no ambiente construído bastante pertinentes. Contudo, deparou-se com que todos os autores são internacionais pois nenhum artigo de autor brasileiro satisfez os critérios de busca.

Será apresentada no Quadro 2, uma síntese dos principais resultados encontrados nesta revisão sistemática, indicando quais foram as principais contribuições das pesquisas para o desenvolvimento desta revisão e para o desenvolvimento do 
Quadro 2 - Síntese dos artigos utilizados nesta RSL (Continua)...................................

\begin{tabular}{|c|c|c|}
\hline \multicolumn{2}{|l|}{ Autores } & Artigos \\
\hline$\underset{z}{z}$ & $\begin{array}{l}\text { Título do } \\
\text { artigo }\end{array}$ & $\begin{array}{l}\text { Bath transfers in older adult congregate } \\
\text { housing residents: Assessing the person- } \\
\text { environment interaction }\end{array}$ \\
\hline 帘 & $\begin{array}{l}\text { Tipo de } \\
\text { pesquisa }\end{array}$ & Análise Transversal \\
\hline$\stackrel{2}{\frac{u}{4}}$ & Participantes & $\begin{array}{l}89 \text { pessoas com } 60 \text { anos ou mais (média de } \\
\text { idade } 82,6 \text { anos). }\end{array}$ \\
\hline 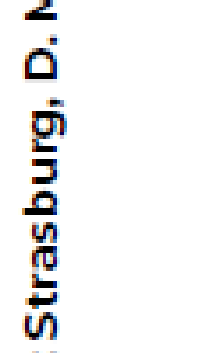 & Objetivos & $\begin{array}{l}\text { Examinar a Utilização de Recursos Ambientais } \\
\text { (URA) e os tipos e prevalência de dificuldades de } \\
\text { desempenho durante uma transferência de } \\
\text { banho gravada em vídeo e para determinar as } \\
\text { características pessoais associadas ao URA total } \\
\text { e as dificuldades de desempenho. }\end{array}$ \\
\hline 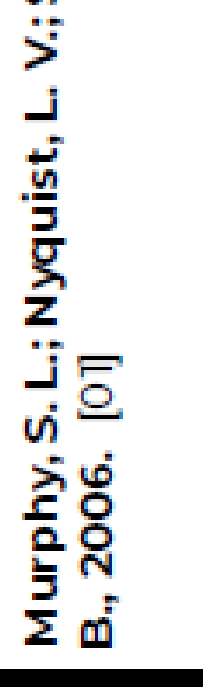 & Resultados & $\begin{array}{l}\text { URA inseguros e dificuldades de desempenho } \\
\text { eram comuns em adultos idosos que tomavam } \\
\text { banho de maneira independente. A dificuldade } \\
\text { de transferência de banho autorreferida parece } \\
\text { ser um bom indicador de URA total alto e pode } \\
\text { ser usada como uma questão de triagem para } \\
\text { clínicos. Estratégias importantes para reduzir o } \\
\text { URA não seguro e aumentar a eficácia da } \\
\text { prevenção das quedas incluem a remoção de } \\
\text { portas de vidro deslizantes e o treinamento de } \\
\text { adultos mais velhos em técnicas de } \\
\text { transferência seguras. }\end{array}$ \\
\hline 宅 & $\begin{array}{l}\text { Título do } \\
\text { artigo }\end{array}$ & $\begin{array}{l}\text { Person-environment fit predicts falls in older } \\
\text { adults better than the consideration of } \\
\text { environmental hazards only }\end{array}$ \\
\hline 뜬 & $\begin{array}{l}\text { Tipo de } \\
\text { pesquisa }\end{array}$ & $\begin{array}{l}\text { Estudo de levantamento com coleta de dados } \\
\text { em visitas domiciliares. }\end{array}$ \\
\hline$\ddot{v} \tilde{i}$ & Participantes & $\begin{array}{l}834 \text { pessoas com idades entre } 75 \text { e } 89 \text { anos } \\
\text { (mediana de idade } 83 \text { anos). }\end{array}$ \\
\hline 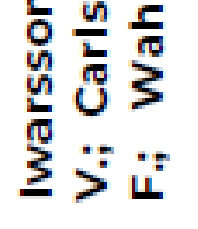 & Objetivos & $\begin{array}{l}\text { Testar as hipóteses de que a consideração } \\
\text { empírica do ajuste objetivo pessoa-ambiente no } \\
\text { ambiente doméstico é um forte preditor de } \\
\text { quedas internas entre adultos mais velhos do }\end{array}$ \\
\hline
\end{tabular}

que a avaliação apenas de barreiras ambientais e que os aspectos percebidos das atividades no ambiente doméstico têm um papel nas quedas.

Os resultados sugerem que grande parte da inconclusividade dos dados na relação entre riscos ambientais e quedas na literatura apresentados anteriormente pode ser devido à

Resultados negligência da avaliação do ajuste pessoaambiente. A eficácia das intervenções ambientais com base na noção de ajuste pessoa-ambiente, em comparação com as listas de verificação de risco domésticas tradicionais, ainda precisa ser testada.

Quadro 2 - Síntese dos artigos utilizados nesta RSL (Continuação)

\begin{tabular}{|c|c|c|}
\hline$\ddot{\underline{Y}}$ & $\begin{array}{l}\text { Título do } \\
\text { artigo }\end{array}$ & $\begin{array}{l}\text { Social Isolation and Physical Barriers in the } \\
\text { Houses of Stroke Survivors in Rural China }\end{array}$ \\
\hline 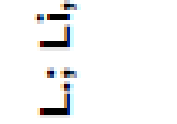 & $\begin{array}{l}\text { Tipo de } \\
\text { pesquisa }\end{array}$ & Análise Transversal \\
\hline วే & Participantes & $\begin{array}{l}818 \text { pessoas com } 60 \text { anos ou mais (média de idade } \\
69 \text { anos). }\end{array}$ \\
\hline 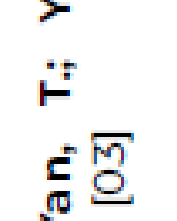 & Objetivos & $\begin{array}{l}\text { Descrever as barreiras domiciliares e o isolamento } \\
\text { social de sobreviventes de AVC nas áreas rurais da } \\
\text { China e explorar quais barreiras domésticas estão } \\
\text { associadas ao isolamento social. }\end{array}$ \\
\hline 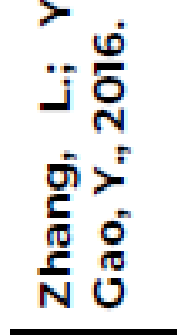 & Conclusões & $\begin{array}{l}\text { Muitas barreiras existem nas casas de } \\
\text { sobreviventes de AVC na China rural. Alguns deles } \\
\text { estão relacionados ao isolamento social. Eliminar } \\
\text { ou diminuir as barreiras domésticas pode ser uma } \\
\text { abordagem viável e eficaz para reduzir o } \\
\text { isolamento social. }\end{array}$ \\
\hline 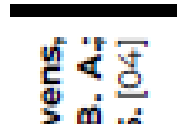 & $\begin{array}{l}\text { Título do } \\
\text { artigo }\end{array}$ & $\begin{array}{l}\text { A self-assessment tool was reliable in identifying } \\
\text { hazards in the homes of elders }\end{array}$ \\
\hline 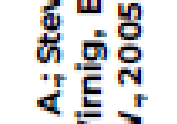 & $\begin{array}{l}\text { Tipo de } \\
\text { pesquisa }\end{array}$ & Análise Transversal \\
\hline 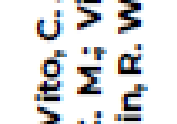 & Participantes & $\begin{array}{l}52 \text { mulheres idosas com idades entre } 67 \text { e } 97 \text { anos } \\
\text { (média de idade } 78,5 \text { anos) }\end{array}$ \\
\hline 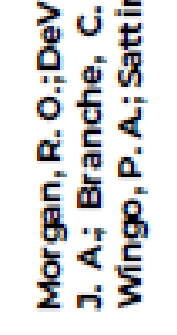 & Objetivos & $\begin{array}{l}\text { As quedas são uma das principais causas de } \\
\text { lesóes fatais e não fatais, particularmente entre os } \\
\text { idosos. Um instrumento confiável para a } \\
\text { autoavaliação dos riscos de quedas em } \\
\text { residências facilitaria a triagem do risco de } \\
\text { quedas. Este estudoexaminou confiabilidade da }\end{array}$ \\
\hline
\end{tabular}




\begin{tabular}{|c|c|c|}
\hline & \multirow[b]{2}{*}{ Resultados } & $\begin{array}{l}\text { autoavaliação dos riscos de quedas } \\
\text { residências em mulheres idosas. }\end{array}$ \\
\hline & & $\begin{array}{l}\text { Esta lista de checagem domiciliar é um passo } \\
\text { importante em direção a um instrumento } \\
\text { confiável de autorrelato para medir os riscos de } \\
\text { quedas em casa. A autoavaliação parece ser um } \\
\text { método confiável para avaliar muitos riscos } \\
\text { presumíveis de cair em casa. No entanto, nossos } \\
\text { resultados levantam questôes sobre a avaliação } \\
\text { confiável dos riscos de tropeço. }\end{array}$ \\
\hline \multirow{5}{*}{ 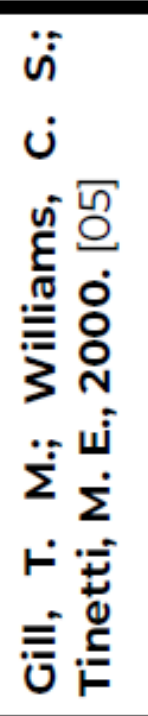 } & $\begin{array}{l}\text { Título do } \\
\text { artigo }\end{array}$ & $\begin{array}{l}\text { Environmental hazards and the risk of } \\
\text { nonsyncopal falls in the homes of community- } \\
\text { living older persons }\end{array}$ \\
\hline & $\begin{array}{l}\text { Tipo de } \\
\text { pesquisa }\end{array}$ & Estudo prospectivo de coorte \\
\hline & Participantes & $\begin{array}{l}1088 \text { pessoas com } 72 \text { anos ou mais (média de } \\
\text { idade } 79,6 \text { anos). }\end{array}$ \\
\hline & Objetivos & $\begin{array}{l}\text { O objetivo deste estudo foi determinar se os riscos } \\
\text { ambientais aumentam o risco de quedas não } \\
\text { sinápticas nos domicílios de idosos que vivem na } \\
\text { comunidade. }\end{array}$ \\
\hline & Conclusão & $\begin{array}{l}\text { Nossos achados não suportam uma associação } \\
\text { entre riscos ambientais e quedas não sincopais. }\end{array}$ \\
\hline \multirow{6}{*}{ 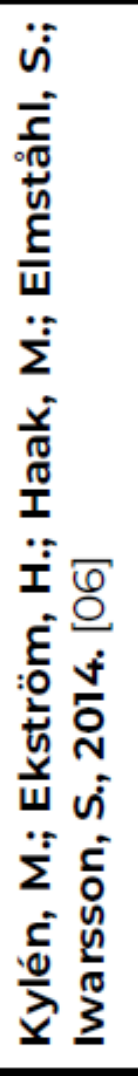 } & $\begin{array}{l}\text { Título do } \\
\text { artigo }\end{array}$ & $\begin{array}{l}\text { Home and health in the third age - } \\
\text { Methodological background and descriptive } \\
\text { findings }\end{array}$ \\
\hline & $\begin{array}{l}\text { Tipo de } \\
\text { pesquisa }\end{array}$ & Análise Transversal \\
\hline & Participantes & $\begin{array}{l}371 \text { pessoas com idades entre } 67 \text { e } 70 \text { anos (média } \\
\text { de idade } 68,4 \text { anos). }\end{array}$ \\
\hline & Contexto & $\begin{array}{l}\text { A compreensão da complexa relação entre o } \\
\text { ambiente doméstico, o bem-estar e o } \\
\text { funcionamento diário na terceira idade é } \\
\text { atualmente fraca. }\end{array}$ \\
\hline & Objetivos & $\begin{array}{l}\text { O objetivo deste artigo é apresentar os } \\
\text { antecedentes metodológicos do Estudo "Casa e } \\
\text { Saúde na Terceira Idade" e descrever uma } \\
\text { amostra de homens e mulheres em relação à sua } \\
\text { situação domiciliar e de saúde. }\end{array}$ \\
\hline & Resultados & $\begin{array}{l}\text { A maioria dos participantes estava com boa saúde } \\
\text { e com poucas limitações funcionais. Barreiras } \\
\text { ambientais foram encontradas em todos os lares } \\
\text { investigados; a maioria foi encontrada na área de }\end{array}$ \\
\hline
\end{tabular}

cozinha e higiene. As barreiras ambientais eram mais comuns em residências multifamiliares do que em residências unifamiliares. Este estudo aumentará nosso conhecimento sobre a dinâmica do lar e da saúde entre pessoas na terceira idade.

\begin{tabular}{|c|c|c|}
\hline 꽁응 & $\begin{array}{l}\text { Título do } \\
\text { artigo }\end{array}$ & $\begin{array}{l}\text { Older adults' attitudes toward home } \\
\text { modifications for fall prevention } \\
\end{array}$ \\
\hline$\ddot{\dot{\alpha}}$ & $\begin{array}{l}\text { Tipo de } \\
\text { pesquisa }\end{array}$ & Entrevistas semiestruturadas \\
\hline i் & Participantes & $\begin{array}{l}10 \text { pessoas com idades entre } 60 \text { e } 92 \text { anos (apenas } \\
1 \text { pessoa tinha } 60 \text { anos, todas as outras tinham } 78 \\
\text { anos ou mais). }\end{array}$ \\
\hline 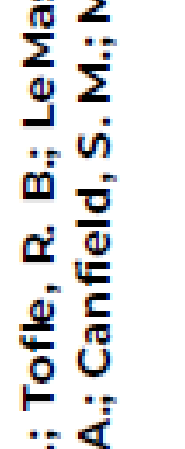 & Objetivos & $\begin{array}{l}\text { Para determinar as atitudes dos idosos em } \\
\text { relação a riscos de queda e modificação domiciliar } \\
\text { para alterar esses riscos, avaliamos as casas dos } \\
\text { participantes e enamos entrevistas } \\
\text { semiestruturadas para explorar o histórico de } \\
\text { quedas e lesões, o risco de queda e as atitudes } \\
\text { para modificar sua residência para reduzir o risco } \\
\text { de queda. }\end{array}$ \\
\hline $\begin{array}{l}\dot{\Sigma} \\
\dot{\Sigma} \\
\dot{\Sigma}\end{array}$ & Resultados & $\begin{array}{l}\text { Geralmente, os participantes não viam suas } \\
\text { quedas como um problema, não acreditavam } \\
\text { que precisassem de ajuda para evitar quedas, não } \\
\text { acreditavam que modificar suas casas evitasse } \\
\text { quedas e não gostavam que outros lhes } \\
\text { dissessem para mudar de casa. Implicações do } \\
\text { estudo sugerem que as estratégias de prevenção } \\
\text { de quedas estão intrinsecamente ligadas ao } \\
\text { relacionamento do idoso com a própria casa, } \\
\text { desafiando a utilidade de simples listas de } \\
\text { verificação de riscos domésticos. }\end{array}$ \\
\hline
\end{tabular}

Quadro 2 - Síntese dos artigos utilizados nesta RSL (Continuação).........................

\begin{tabular}{|c|c|c|}
\hline \multirow{3}{*}{ 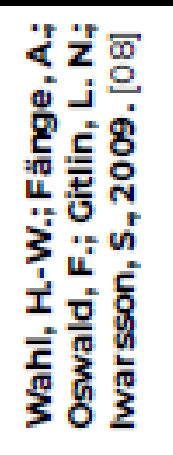 } & $\begin{array}{l}\text { Título do } \\
\text { artigo }\end{array}$ & $\begin{array}{l}\text { The home environment and disability-related } \\
\text { outcomes in aging individuals: What is the } \\
\text { empirical evidence? }\end{array}$ \\
\hline & $\begin{array}{l}\text { Tipo de } \\
\text { pesquisa }\end{array}$ & Revisão Sistemática \\
\hline & Objetivos & $\begin{array}{l}\text { Com base no modelo de processo de incapacidade } \\
\text { e no conceito de ajuste pessoa - ambiente (ajuste }\end{array}$ \\
\hline
\end{tabular}




\begin{tabular}{|c|c|}
\hline & $\begin{array}{l}\text { p-a), este artigo de revisão examina duas questões } \\
\text { críticas sobre o papel dos ambientes domésticos: } \\
\text { (a) Quais são as evidências recentes que sustentam } \\
\text { um relacionamento entre os ambientes } \\
\text { domésticos e os resultados relacionados à } \\
\text { deficiência? e (b) Quais são as evidências recentes } \\
\text { sobre os efeitos das modificações domiciliares nos } \\
\text { desfechos relacionados à deficiência? }\end{array}$ \\
\hline Resultados & $\begin{array}{l}\text { Para a questão de pesquisa } 1 \text {, existem evidências } \\
\text { de uma relação entre os ambientes domésticos e } \\
\text { os desfechos relacionados à incapacidade para } \\
\text { adultos mais velhos, mas são limitados por projetos } \\
\text { transversais e má qualidade de pesquisa. Para a } \\
\text { pergunta de pesquisa } 2 \text {, evidências baseadas em } \\
\text { ensaios clínicos randomizados e controlados } \\
\text { mostram que melhorar o ambiente doméstico } \\
\text { aumenta os resultados de habilidades funcionais, } \\
\text { mas não tanto os resultados relacionados a } \\
\text { quedas. Existem algumas evidências de que os } \\
\text { estudos que usam uma perspectiva de ajuste p-a } \\
\text { resultam em descobertas mais favoráveis do que } \\
\text { os estudos que não usam esse quadro. } \\
\text { Implicações: existem evidências consideráveis que } \\
\text { apoiam o papel dos ambientes domésticos no } \\
\text { processo de incapacitação, mas também há } \\
\text { inconsistências nos achados dos estudos. }\end{array}$ \\
\hline Conclusões & $\begin{array}{l}\text { Pesquisas futuras devem otimizar propriedades } \\
\text { psicométricas de ferramentas de avaliação do } \\
\text { ambiente doméstico explorar o papel de ambas } \\
\text { as características, objetivas e atribuídas, dos } \\
\text { ambientes domésticos para entender a dinâmica } \\
\text { pessoa - ambiente e o seu impacto sobre os } \\
\text { resultados relacionados com a deficiência na } \\
\text { velhice. }\end{array}$ \\
\hline
\end{tabular}

\begin{tabular}{|c|c|c|}
\hline \multirow{5}{*}{ 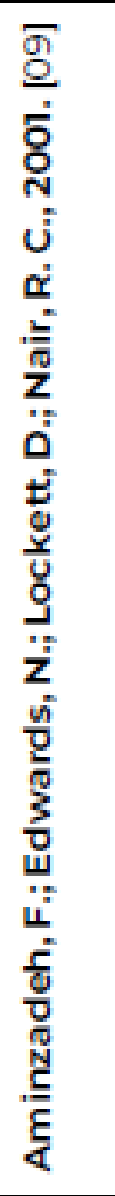 } & $\begin{array}{l}\text { Titulo do } \\
\text { artigo }\end{array}$ & $\begin{array}{l}\text { Utilization of bathroom safety devices, patterns of bathing } \\
\text { and toileting, and bathroom falls in a sample of community }\end{array}$ \\
\hline & $\begin{array}{l}\text { Tipo de } \\
\text { pesquisa }\end{array}$ & Análise Transversal \\
\hline & Participantes & 550 pessoas com 60 anos ou mais (média de idade 73,9 anos) \\
\hline & Objetivos & $\begin{array}{l}\text { Examinar padröes de utilização e aceitabilidade dos } \\
\text { dispositivos de segurança do banheiro, práticas de banho } \\
\text { higiene, e quedas no banheiro em uma amostra de } 550 \\
\text { residentes mais velhos de prédios de apartamentos sem fins } \\
\text { lucrativos em duas regiöes canadenses. }\end{array}$ \\
\hline & Resultados & $\begin{array}{l}\text { Quase um terço dos participantes (32\%) tiveram pelo menos } \\
\text { uma queda no ano anterior ao estudo, dos quais cerca de } 15 \% \\
\text { cairam no banheiro. Mais da metade das quedas do banheirc } \\
\text { foram relacionadas a transferências da banheira ou WC } \\
80 \% \text { resultaram em lesöes leves a graves. A maioria dos } \\
\text { idosos com acesso a dispositivos de segurança no banheirc } \\
\text { os usava regularmente e os considerava úteis. No entanto } \\
\text { muitos participantes também contavam com suportes } \\
\text { perigosos para facilitar as atividades de banho e higiene. As } \\
\text { implicaçōes dos resultados estão relacionadas ac } \\
\text { planejamento de políticas, design de produtos } \\
\text { desenvolvimento de padrōes e educaçäo pública. }\end{array}$ \\
\hline \multirow{5}{*}{ 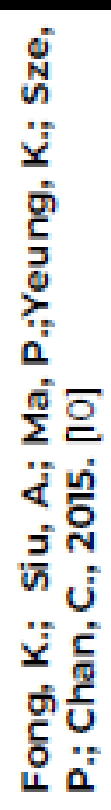 } & $\begin{array}{l}\text { Titulo do } \\
\text { artigo }\end{array}$ & $\begin{array}{l}\text { Domiciliary environmental risk factors for accidental falls } \\
\text { among community-living older persons: A prospective } 12 \\
\text { month study }\end{array}$ \\
\hline & $\begin{array}{l}\text { Tipo de } \\
\text { pesquisa }\end{array}$ & Análise Transversal \\
\hline & Participantes & 592 pessoas com 65 anos ou mais (média de idade 75 anos). \\
\hline & Objetivos & $\begin{array}{l}\text { Este estudo examinou os fatores de risco ambientais } \\
\text { domiciliares atribuiveis a quedas acidentais entre pessoas } \\
\text { idosas vivendo na comunidade que moram em prédios altos } \\
\text { em Hong Kong. }\end{array}$ \\
\hline & Resultados & $\begin{array}{l}\text { Este estudo confirma a existência de uma diferença nos } \\
\text { fatores de risco ambientais entre os que caem e os que nā́ } \\
\text { caem nos edificios de apartamentos, e os resultados poder } \\
\text { ser generalizados para outros ambientes domiciliares para } \\
\text { pessoas idosas da comunidade na maioria das cidades } \\
\text { urbanas. }\end{array}$ \\
\hline
\end{tabular}




\begin{tabular}{|c|c|c|}
\hline \multirow{6}{*}{ 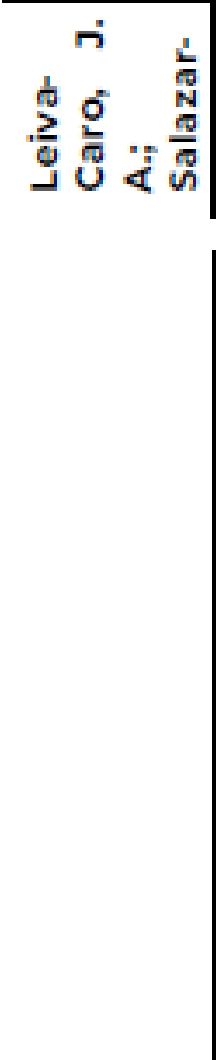 } & $\begin{array}{l}\text { Titulo do } \\
\text { artigo }\end{array}$ & $\begin{array}{l}\text { Connection between competence, usability, environment } \\
\text { and risk of falls in elderly adults }\end{array}$ \\
\hline & $\begin{array}{l}\text { Tipo de } \\
\text { pesquisa }\end{array}$ & Estudo descritivo correlacional \\
\hline & Participantes & 123 pessoas com 70 anos ou mais (média de idade 77,5 anos). \\
\hline & Objetivos & $\begin{array}{l}\text { Determinar a relaçäo entre competência, usabilidade e o } \\
\text { ambiente com o risco de quedas dos individuos idosos. }\end{array}$ \\
\hline & Resultados & $\begin{array}{l}42,0 \% \text { dos idosos apresentaram quedas, sendo maior a } \\
\text { prevalência nas mulheres e no grupo de } 70-75 \text { anos. Como } \\
\text { fator de risco de quedas, foram encontrados oambiente físico } \\
\text { da moradia, caminhada e usabilidade. Encontrou-se relação } \\
\text { negativa entre usabilidade com sintomas depressivos, saúde } \\
\text { cognitiva, equilibrio, caminhada, ambiente social e físico } \\
\text { p<0,05; e forte correlação positiva entre caminhada e } \\
\text { equilibrio p<0,05. }\end{array}$ \\
\hline & Conclusäo & $\begin{array}{l}\text { O estudo contribui para melhor compreensäo formal do } \\
\text { fenômeno das quedas ao encontrar relação entre a } \\
\text { usabilidade com o risco de quedas, e, também, com outras } \\
\text { variáveis que se relacionam com as quedas }\end{array}$ \\
\hline \multirow{5}{*}{ 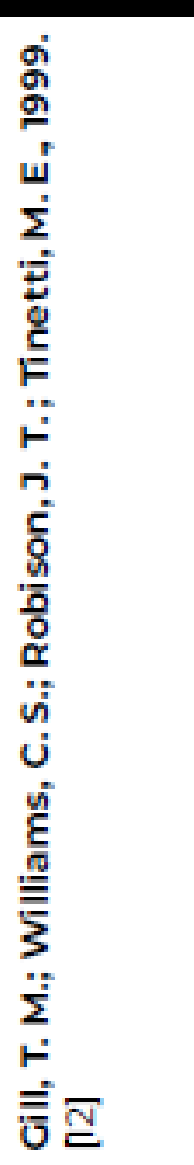 } & $\begin{array}{l}\text { Titulo do } \\
\text { artigo }\end{array}$ & $\begin{array}{l}\text { A population-based study of environmental hazards in the } \\
\text { homes of older persons }\end{array}$ \\
\hline & $\begin{array}{l}\text { Tipo de } \\
\text { pesquisa }\end{array}$ & Estu \\
\hline & Participantes & 1103 pessoas com 72 anos ou mais (média de idade 79,6 anos). \\
\hline & Objetivos & $\begin{array}{l}\text { Nossos objetivos especificos foram estimar a prevalência de } \\
\text { riscos ambientais com base na populaçäo e determinar se a } \\
\text { prevalência desses perigos varia de acordo com o tipo de } \\
\text { alojamento ou com o nivel de incapacidade das AVDs. }\end{array}$ \\
\hline & Resultados & $\begin{array}{l}\text { A estratégia de amostragem permitiu comparar a } \\
\text { prevalência de riscos potenciais entre moradia restrita por } \\
\text { idade e moradia comunitária. Embora a maioria dos riscos } \\
\text { tenha sido menos prevalente em moradias restritas por idade } \\
\text { do que em moradias comunitárias, vários riscos foram } \\
\text { surpreendentemente comuns em moradias com idade } \\
\text { limitada, o que muitas vezes atende a uma subpopulação } \\
\text { mais frágil de idosos em idade de convivência. Com base } \\
\text { nesses achados, seria um erro presumir que a moradia } \\
\text { restrita por idade seja inerentemente segura e desprovida de } \\
\text { riscos potenciais. }\end{array}$ \\
\hline
\end{tabular}

\begin{tabular}{|c|c|c|}
\hline \multirow{5}{*}{ 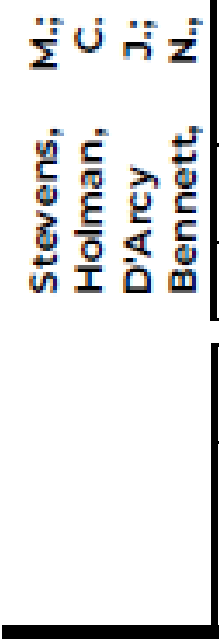 } & $\begin{array}{l}\text { Titulo do } \\
\text { artigo }\end{array}$ & $\begin{array}{l}\text { Preventing Falls in Older People: Impact of an Intervention } \\
\text { to Reduce Environmental }\end{array}$ \\
\hline & $\begin{array}{l}\text { Tipo de } \\
\text { pesquisa }\end{array}$ & Ensaio experimental \\
\hline & Participantes & 570 pessoas com 70 anos ou mais. \\
\hline & Objetivos & $\begin{array}{l}\text { Avaliar o impacto de uma intervenção para reduzir os riscos } \\
\text { de queda nas casas dos idosos. }\end{array}$ \\
\hline & Resultados & $\begin{array}{l}\text { Todas as casas tiveram pelo menos um risco de queda. } \\
\text { Alguns foram mais prevalentes. A intervençăo foi associada a } \\
\text { uma pequena, mas significativa reduçăo em quatro dos cinco } \\
\text { riscos mais prevalentes. }\end{array}$ \\
\hline \multirow{5}{*}{ 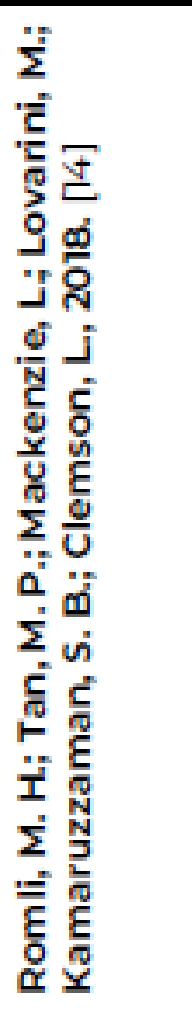 } & $\begin{array}{l}\text { Titulo do } \\
\text { artigo }\end{array}$ & $\begin{array}{l}\text { Factors associated with home hazards: Findings from the } \\
\text { Malaysian Elders Longitudinal Research study }\end{array}$ \\
\hline & $\begin{array}{l}\text { Tipo de } \\
\text { pesquisa }\end{array}$ & Análise Transversal \\
\hline & Participantes & $\begin{array}{l}\text { 1489 pessoas com } 60 \text { anos ou mais (média de idade } 68,7 \\
\text { anos). }\end{array}$ \\
\hline & Objetivos & $\begin{array}{l}\text { Estudos prévios investigaram os riscos domiciliares como } \\
\text { fator de risco para quedas sem considerar fatores associados } \\
\text { à presença de riscos residenciais. O presente estudo teve } \\
\text { como objetivo determinar os padröes de riscos domésticos } \\
\text { entre os habitantes da Malásia residentes na comunidade } \\
\text { urbana e identificar os fatores que contribuem para os } \\
\text { perigos domésticos. }\end{array}$ \\
\hline & Conclusöes & $\begin{array}{l}\text { Este estudo fornece evidências de que os riscos domésticos } \\
\text { são um produto da interaçäo da funçăo do individuo dentro } \\
\text { de seu ambiente doméstico. Os riscos também são } \\
\text { influenciados por fatores socioculturais eambientais locais. A } \\
\text { relação entre os riscos domésticos e quedas parece ser } \\
\text { complexa e merece uma avaliaçäo mais aprofundada. }\end{array}$ \\
\hline
\end{tabular}

Fonte: Elaborado pelos autores (2018)

\subsection{CARACTERÍSTICAS DOS ESTUDOS INCLUÍDOS E DESCOBERTAS}

Após a análise dos 14 estudos, evidencia-se um avanço das pesquisas ao longo dos anos, pois após uma concentração inicial de publicações entre 1999 e 2001 com quatro publicações, houve uma nova concentração entre 2009 e 2010 também com quatro publicações. E a partir de 2014, uma retomada, com outras quatro publicações. Também se observa que os estudos estão localizados em centros de pesquisa nos 
Estados Unidos da América (cinco publicações), Suécia (duas publicações), China (duas publicações), e, Alemanha, Austrália, Canadá, Malásia e México (com uma publicação cada). Uma possível causa para esse foco nos Estados Unidos da América pode ser o fato dos artigos serem publicados no idioma inglês ou mesmo de estarem relacionados a grupos de estudos de pesquisadores americanos pioneiros nesse tema de pesquisa. O grupo formado por pesquisadores europeus também é bastante atuante, principalmente os pesquisadores suecos.

A maioria dos estudos (11 em 14) foi do tipo análise transversal, mas houve uma revisão sistemática e dois estudos prospectivos de coorte. Ambos os estudos longitudinais tiveram a duração de 3 anos. No que tange às áreas de conhecimento percebem-se as seguintes áreas publicando estudos sobre o tema: saúde, design, arquitetura, psicologia e gerontologia. Também chama a atenção o número expressivo de artigos publicados (doze publicações) em periódicos relacionados as áreas da saúde (geriatria e enfermagem) e a gerontologia, se comparado ao total das publicações. o que denota a necessidade de um maior número de pesquisas e publicações, por parte de ergonomistas, arquitetos e designers, relacionadas diretamente ao tema de pesquisa desse estudo.

Observa-se que o conjunto das pesquisas tem como foco a intervenção no ambiente doméstico, particularmente com o objetivo de reduzir a incapacidade e a perda de autonomia à medida que as pessoas envelhecem. $O$ argumento é que a melhoria do ambiente físico-social por meio de modificações domésticas deve ter um impacto positivo na qualidade de vida na terceira idade (AMINZADEH et al., 2001; FONC et al., 2015; ZHANG et al., 2016). Os resultados das pesquisas, como descrito acima, denotam que esse argumento abrangente seria válido, mas, como se pode constatar a maioria das pesquisas tem sido transversal.

Esta revisão da literatura sobre as evidências disponíveis sobre a relação idoso-ambiente doméstico revelou 13 investigações originais e 1 artigo de revisão. Os resultados predominantes incluíram as atividades de avaliação da vida diária e acidentes domésticos. Uma descoberta importante desta revisão é que, analisando os estudos em conjunto, há evidências de que modificações no ambiente doméstico são capazes de reduzir os resultados relacionados as atividades de vida diária, com a maioria dos estudos relatando pelo menos evidências parcialmente favoráveis (FONC et al., 2015; IWARSSON et al., 2009; LEIVA-CARO et al., 2015; KRUSE et al., 2010; KYLÉN et al., 2014; MORGAN et al., 2005; ROMLI et al., 2018; STEVENS; HOLMAN; BENNETT, 2001; ZHANG et al., 2016). Vários estudos indicam claramente que a eliminação dos riscos ao ambiente doméstico é capaz de reduzir a taxa de quedas em idosos e essa evidência está alinhada com compilações anteriores da literatura (WAHL et al., 2009).

A faixa etária dos participantes em todos os estudos foi de 60 a 97 anos ou mais, com mais da metade dos estudos incluindo aqueles com 65 anos ou mais. O tamanho da amostra dos estudos variou muito, desde amostras com $\mathrm{N}=10$ até amostras com $\mathrm{N}=1.489$. Apenas $28,6 \%$ dos estudos ( 4 de 14 ), consideraram pessoas idosas com capacidade funcional limitada (por exemplo, usuários de cadeira de rodas, ou usuário que utilizam andadores ou bengala), porém nenhum estudo incluído nesta revisão teve participantes com transtornos cognitivos. Pelo fato da maioria dos estudos terem participantes considerados idosos velhos, é possível que a maioria estivesse em risco de declínio funcional. Idade, sexo e educação foram considerados variáveis de confusão em dois $(14,3 \%)$ dos estudos. Outros itens que foram levantados, estão descritos na tabela 2 e serão analisados na sequência.
Tabela 2 - Itens referentes aos riscos domésticos abordados nos artigos.

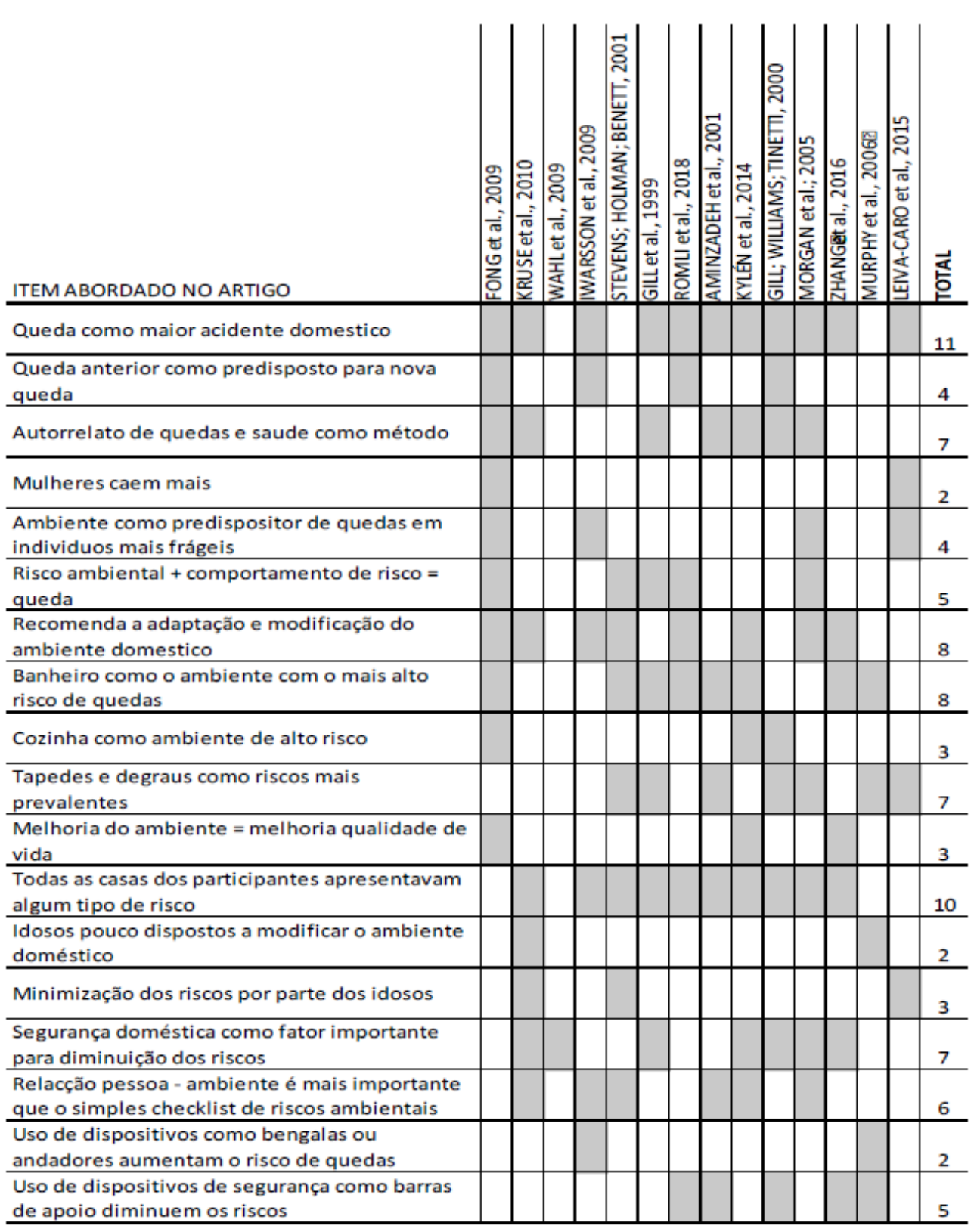

Fonte: Elaborado pelos autores (2018)

A ampla maioria, 11 dos 14 estudos (78,6\%), considerou a queda como o maior acidente doméstico e o que causa maiores sequelas aos idosos. O medo de cair é um fato apontado pelos idosos e está presente nestes mesmos estudos. Quatro deles $(28,6 \%)$ apontaram uma queda anterior como fator predispositor de uma nova caída. Além disso, 14,3\% dos estudos afirmam que as mulheres caem mais do que os homens. Em quatro $(28,6 \%)$ encontrou-se evidências de riscos ambiente domiciliar como outro fator predispositor de quedas em idosos frágeis.

O banheiro com $57,1 \%(8 / 14)$ e a cozinha com $21,4 \%$ (3/14) dos estudos foram os ambientes mais citados em termos de risco doméstico. Os tapetes soltos, degraus irregulares e sem tratamento antiderrapante, bem como cabos soltos e outros objetos no piso foram citados por $50 \%$ dos artigos. A quase totalidade dos estudos afirmou 
que haviam riscos ambientas em quase $100 \%$ das casas dos participantes .Porém três $(21,4 \%)$ dos autores dizem que os idosos minimizam os seus riscos de queda devido aos perigos domésticos e apesar da segurança doméstica ser um fator preponderante por parte dos idosos (citada em 50\% dos estudos) alguns não estão dispostos a fazem modificações em suas casas (segundo 14\% dos artigos).

\section{RESULTADOS E DISCUSSÃO}

Fong et al. (2015) em seu estudo, ao que se refere aos fatores extrínsecos, encontraram diferenças significativas nos ambientes domésticos entre os grupos de pessoas caídoras e não caídoras, nas áreas de risco de queda. Estas diferenças estavam por exemplo em questões de acessibilidade e altura de equipamentos e acessórios. Havia diferenças relativas a lugares de sentar, banheiros e cozinhas, e também com as características de escadas e degraus, bem como a inexistência de corrimãos (FONG et al., 2015).

Fatores ambientais domésticos que se acredita serem possíveis riscos de queda (por exemplo, a presença de tapetes, piso escorregadio, degraus, falta de barras de apoio) são amplamente prevalentes (AMINZADEH et al., 2001; GILL et al., 1999; GILL; WILLIAMS; TINETTI, 2000; KYLÉN et al., 2014; MORGAN et al., 2005; MURPHY et al., 2006; STEVENS; HOLMAN; BENNETT, 2001) e foram associados com maior risco de quedas (MORGAN et al., 2005), principalmente em indivíduos mais frágeis (IWARSSON et al., 2009). Entre idosos indivíduos com histórico de queda, intervenções para reduzir os perigos de quedas em casa mostraram de fato, reduzir o número das quedas (FONG et al., 2015; IWARSSON et al., 2009; LEIVA-CARO et al., 2015; MORGAN et al., 2005)

Dificuldades com atividades de banho, quedas no banheiro e medo de cair são ocorrências comuns entre muitos idosos, é o que afirmam Aminzadeh et al. (2001) Os autores destacam os benefícios potenciais de dispositivos de segurança bem projetados para melhorar a segurança e independência desta parcela da população, pois cerca de um terço dos idosos relataram que faziam restrições em seus hábitos de higiene devido ao medo de cair.

Segundo Romli et al. (2018) as quedas só podem ocorrer se as exigências funcionais do ambiente doméstico impostas pelos perigos domésticos excederem a capacidade de equilíbrio da pessoa idosa. E prosseguem dizendo que o gerenciamento de riscos domiciliares por meio de avaliação e modificação reduziu significativamente os riscos domésticos e as quedas.

Uma pessoa deve ter um alto nível de competência para lidar eficazmente com um ambiente com altas demandas. Geralmente as pessoas mais velhas não vão cair se forem capazes de se protegerem se adaptando ao ambiente, como escadas íngremes. Mas a pessoa pode ser vulnerável a quedas e o ambiente pode se tornar arriscado para aqueles com deterioração das condições de saúde, como o declínio do equilíbrio e da mobilidade, bem como para aqueles com histórico de quedas (FONG et al., 2015). Os autores prosseguem afirmando que "adultos mais velhos com uma marcha instável podem cair quando não conseguem mais lidar com a subida de um lance de escadas devido à perda de capacidade de manter o equilíbrio, o que significa que não podem responder adaptativamente ao ambiente".

Desta forma, modificações no ambiente doméstico podem ser muito úteis para suscitar um ambiente positivo e reduzir o risco de quedas. Até mesmo modificações caseiras simples podem aumentar o espaço de atividades de um indivíduo com uma deficiência (ZHANG et al., 2016). Quando as pessoas sentem que estão no controle de suas atividades e residência, elas podem estar mais propensas a procurar formas de reduzir o risco de cair (KRUSE et al., 2010). Porém, a literatura constatou, segundo Aminzadeh et al. (2001), inúmeras barreiras com base no usuário, no dispositivo e no ambiente para o uso de dispositivos de segurança. E isto poderia ocorrer, devido à falta de conhecimento sobre a existência e o valor dos dispositivos, a negação da necessidade do uso, o medo do estigma da velhice, o custo e até mesmo a falha do dispositivo. Além disso, argumentam os autores que os padrões de acessibilidade são baseados nas necessidades das pessoas com deficiência de todas as idades, o que pode não ser apropriado para a população idosa. (AMINZADEH et al., 2001)

Também podemos observar várias pesquisas o resultado encontrado de que o ambiente físico da moradia se relaciona diretamente com o risco de quedas corroborando afirmações de que os perigos no lar aumentam o risco de quedas. Mesmo que o número de barreiras da moradia seja baixo, é importante considerar o tipo de barreira da moradia e as características pessoais do idoso em termos de competência, visto que a queda é produzida no momento de interação do idoso com o ambiente (LEIVA-CARO et al., 2015). De acordo com Wahl et al. (2009) é necessário considerar e medir, tanto o ambiente familiar objetivo quanto o percebido, para compreender o quadro completo da dinâmica pessoa-ambiente e seu impacto nos resultados relacionados à deficiência.

\section{CONCLUSÃO}

De acordo com o objetivo desta pesquisa, que consistiu em identificar publicações referentes a relação entre idosos, ambiente construído domiciliar e fatores de risco relativos a quedas no ambiente doméstico, é possível inferir que a pesquisa sobre a relação pessoa-ambiente construído pode trazer significativas contribuições para o processo de avaliação dos riscos domésticos e com uma melhor tomada de decisão quanto as modificações a serem adotadas no ambiente construído domiciliar. Pois, embora haja consenso de que o ambiente físico tem o potencial para impor restrições significativas no fim da vida, ele também pode aumentar as oportunidades para envelhecer bem, como novas soluções de habitação e novas tecnologias apoiar competências em declínio.

Diversos autores tratam sobre a temática do envelhecimento e os perigos domésticos. Porém, o que se observa é que parte das publicações analisadas ainda têm seu foco na avaliação do espaço físico apenas, não considerando a interação das pessoas idosas com o ambiente construído doméstico. Segundo ROMLI et al. (2018), essa abordagem unilateral que não leva em conta as características dos indivíduos mais velhos e de como ocorre a interação pessoa-ambiente, pode tornar o ambiente doméstico mais perigoso.

Então, apesar dos riscos domésticos tornarem as quedas "acidentes a espera de suceder", as modificações na segurança doméstica estão intimamente ligadas ao significado da casa como um tipo de autoidentidade da pessoa, principalmente no caso dos idosos, e é preciso considerar isto fortemente. Ainda precisamos aprender muito mais sobre a questão das capacidades individuais e a relação de pertencimento para entender que tipo de intervenções são mais adequadas a quais tipos de idosos, um desafio que exige combinações de esforços de pesquisa quantitativa e qualitativa. Por fim, concluiu-se que a temática ainda não é muito explorada no Brasil, já que a 
maioria dos autores encontrados nas bases teóricas são de outros países. Clara também é a necessidade de maiores aprofundamentos quanto a pesquisas nesta área.

\section{REFERÊNCIAS}

AMINZADEH, F. et al. Utilization of bathroom safety devices, patterns of bathing and toileting, and bathroom falls in a sample of community living older adults. Technology and Disability, v. 13, n. 2, p. 95-103, 2001.

BESTETTI, M. L. T. Habitação para idosos. O trabalho do arquiteto, arquitetura e cidade. 2006. 184f. Tese (Doutorado). Faculdade de Arquitetura e Urbanismo, Universidade de São Paulo, São Paulo, 2006.

CONFORTO, E. C.; AMARAL, D. C.; SILVA, S. L. Roteiro para revisão bibliográfica sistemática: aplicação no desenvolvimento de produtos e gerenciamento de projetos. In: 80 Congresso Brasileiro de Gestão de Desenvolvimento de Produtos. Anais... Porto Alegre: 2011

DARÈ, A. C. L. A Percepção do Idoso do Meio Ambiente Doméstico. In: $7^{\circ}$ Congresso Brasileiro de Pesquisa e Desenvolvimento em Design. Anais... Curitiba: 2006

FELIZARDO, K. R. et al. Revisão Sistemática da Literatura em Engenharia de Software. la. ed. Rio de Janeiro: Elsevier, 2017. ISBN: 9788535286410

FONG, K. N.-K. et al. Domiciliary environmental risk factors for accidental falls among community-living older persons: A prospective 12-month study. Healthy Aging Research, v. 4, n. 5, 2015.

GILL, T. M. et al. A population-based study of environmental hazards in the homes of older persons. American Journal of Public Health, v. 89, n. 4, p. 553-556, abr. 1999.

GILL, T. M.; WILLIAMS, C. S.; TINETTI, M. E. Environmental hazards and the risk of nonsyncopal falls in the homes of community-living older persons. Medical Care, v. 38, n. 12, p. 1174-1183, 2000

HAZIN, M. M. V. Os espaços residenciais na percepção dos idosos ativos. 2012 Dissertação (Mestrado) - Universidade Federal de Pernambuco, Recife, 2012.

IWARSSON, S. et al. Person-environment fit predicts falls in older adults better than the consideration of environmental hazards only. Clinical Rehabilitation, v. 23, n. 6, p. 558-567, jun. 2009

JACKO, J. A. et al. Human Factors and Ergonomic Methods. In: SALVENDY, G. (Ed.).Handbook of Human Factors and Ergonomics. 4. ed. Hoboken, New Jersey: John Wiley \& Sons, Inc., 2012. p. 298-329.

KRUSE, R. L. et al. Older adults' attitudes toward home modifications for fall prevention. Journal of Housing for the Elderly, v. 24, n. 2, p. 110-129, 2010.

HFD, v.9, n 18, p. 150-170, dezembro 2020
KYLEN, M. et al. Home and health in the third age - Methodological background and descriptive findings. International Journal of Environmental Research and Public Health, v. 11, n. 7, p. 7060-7080, 2014.

LEIVA-CARO, J. A. et al. Connection between competence, usability, environment and risk of falls in elderly adults. Revista Latino-Americana de Enfermagem, v. 23, n. 6, p. 1139-1148, dez. 2015.

MORGAN, R. O. et al. A self-assessment tool was reliable in identifying hazards in the homes of elders. Journal of Clinical Epidemiology, v. 58, n. 12, p. 1252-1259, 2005.

MURPHY, S. L. et al. Bath transfers in older adult congregate housing residents: Assessing the person-environment interaction. Journal of the American Geriatrics Society, v. 54 n. 8, p. 1265-1270, 2006

OMS - Organização Mundial da Saúde. Guia global: cidade amiga do idoso. (on-line). 2008. Disponível em: http://www.who.int/ageing/GuiaAFCPortuguese.pdf. Acesso em 30 maio 2016

PREISER, W.F.E. Toward Universal Design Evaluation. In: PREISER, W.F.E.; OSTROFF, E. (eds.). Universal Design Handbook, New York, McGraw-Hill, 2001. pp. 9.1-9.18.

ROMLI, M. H. et al. Factors associated with home hazards: Findings from the Malaysian Elders Longitudinal Research study. Geriatrics \& Gerontology International, v. 18, n. 3 , p. 387-395, mar. 2018.

SIMÕES, J. F.; BISPO, R. Design Inclusivo: acessibilidade e usabilidade em produtos, serviços e ambientes. $2^{\mathrm{a}}$ ed. Lisboa: Centro Português de Design, 2006. ISBN 978-972$9445-33-0$

SPIRDUSO, W. W. Dimensões físicas do envelhecimento. Barueri: Manole, 2005.

STAMATO, C. Modelo de Banheiro Domiciliar para Idosos: Uma Abordagem Ergonômica. Dissertação (Mestrado) - Programa de Pós-graduação em Design, Departamento de Artes e Design - Pontifícia Universidade Católica do Rio de Janeiro, 2007.

STEVENS, M.; HOLMAN, C. D. J.; BENNETT, N. Preventing Falls in Older People: Impact of an Intervention to Reduce Environmental Hazards in the Home. Journal of the American Geriatrics Society, v. 49, n. 11, p. 1442-1447, nov. 2001

URTON, M. M. A community home inspection approach to preventing falls among the elderly. Public Health Reports, v. 106, n. 2, p. 192-195, 1991.

WAHL, H.-W. et al. The home environment and disability-related outcomes in aging individuals: What is the empirical evidence? Gerontologist, v. 49, n. 3, p. 355-367, 2009.

ZHANG, L. et al. Social Isolation and Physical Barriers in the Houses of Stroke Survivors in Rural China. Archives of Physical Medicine and Rehabilitation, v. 97, n. 12, p. $2054-$ 2060, 2016.

HFD, v.9, n 18, p. 150-170, dezembro 2020 